\title{
Gravitational waves from neutrino mass and dark matter genesis
}

\author{
Pasquale Di Bari, ${ }^{1}$ Danny Marfatia, ${ }^{2}$ and Ye-Ling Zhou ${ }^{1}$ \\ ${ }^{1}$ School of Physics and Astronomy, University of Southampton, Southampton SO17 1BJ, United Kingdom \\ ${ }^{2}$ Department of Physics and Astronomy, University of Hawaii at Manoa, Honolulu, Hawaii 96822, USA
}

(Received 27 January 2020; revised 15 July 2020; accepted 2 November 2020; published 16 November 2020)

\begin{abstract}
We introduce a model in which the genesis of dark matter (DM) and neutrino masses is associated with a first-order phase transition of a scalar singlet field. During the phase transition a source right-handed neutrino (RHN) acquires a spacetime-dependent mass dynamically, a small fraction of which is converted via resonant oscillations into a very weakly mixed dark RHN which decays to a dark matter RHN with the observed relic abundance. Neutrino masses are generated via a traditional two RHN type-I seesaw between a fourth RHN and the source neutrino. The gravitational waves produced during the phase transition have a peak frequency that increases with the DM mass, and are detectable at future gravitational-wave interferometers for DM masses above $\sim 1 \mathrm{MeV}$. Since the source RHNs are heavier than the electroweak scale, successful leptogenesis is also attainable.
\end{abstract}

DOI: 10.1103/PhysRevD.102.095017

\section{INTRODUCTION}

The nature of dark matter (DM) is one of the longeststanding puzzles in fundamental physics [1]. Although astrophysical and cosmological observations support a solution in terms of a new particle and disfavor alternative explanations in terms of modified gravity or primordial black holes, all efforts to identify the nature of this new particle, with direct, indirect and collider searches, have failed so far. This produces strong constraints on the existing models of DM, either favoring heavy DM particles (with mass above a TeV), light particles (with mass below a $\mathrm{GeV}$ ) or very weakly coupled ones, such as axion-like particles, or some combination thereof. Therefore, a nonthermal production mechanism, quite different from the usual weakly interacting massive particle paradigm, that relies on very small couplings of the DM particle to the thermal bath, is a reasonable possibility to consider.

At the same time extensions of the standard model (SM) should also account for neutrino masses and mixing, and explain the matter-antimatter asymmetry of the Universe. The type-I seesaw mechanism is the most minimal and attractive way to incorporate neutrino masses and mixing and to explain the matter-antimatter asymmetry of the Universe via leptogenesis. It is then quite reasonable to seek unified models of neutrino masses, DM and leptogenesis starting from the type-I seesaw Lagrangian and extended with some new ingredient that also addresses DM.

Published by the American Physical Society under the terms of the Creative Commons Attribution 4.0 International license. Further distribution of this work must maintain attribution to the author(s) and the published article's title, journal citation, and DOI. Funded by SCOAP ${ }^{3}$.
An example of this kind was proposed in Ref. [2] in which one (dark) right-handed neutrino (RHN) has vanishing Yukawa couplings and therefore does not contribute to neutrino masses and mixing. However, it mixes with the other (source) RHNs and to the standard model Higgs through the five-dimensional nonrenormalizable operator $\left(\lambda_{I J} / \Lambda\right) \Phi^{\dagger} \Phi \overline{N_{R I}^{c}} N_{R J}$ [3]. Consequences of these interactions have been studied in Refs. [4,5].

In this paper we introduce a novel mechanism for the production of RHN DM that relies on a first-order phase transition of a scalar singlet field. It has been proposed that the variation of couplings in the Weinberg operator during a phase transition can provide a new way to implement leptogenesis [6]. Here we show that a phase transition can induce an efficient conversion of source RHNs into a dark RHN species that decays prior to the onset of big bang nucleosynthesis, into a lighter dark RHN species that plays the role of DM. The process is compatible with a standard cosmological history. A strong first-order phase transition is required and an associated production of gravitational waves (GWs) is expected [7-10]. In fact the spectrum of GWs is related to the properties of the DM particle in a way that provides an interesting signature of the model.

Several works have proposed a more or less direct link of DM genesis to a phase transition [7,11-21], some of which also predict a detectable GW spectrum. Additionally, in our scenario there is also an important link with neutrino masses and leptogenesis.

\section{THE MODEL}

In addition to the SM particle content we have four RHNs $N_{\text {DM }}, N_{\mathrm{D}}, N_{1}, N_{2}$ and a new complex scalar field $\eta$ 
which may be associated with the breaking of a flavor symmetry. The two dark RHNs, $N_{\mathrm{DM}}$ and $N_{\mathrm{D}}$, are neutral gauge singlets which acquire a Majorana mass $M_{\mathrm{DM}}$ and $M_{\mathrm{D}}$ respectively, with $M_{\mathrm{DM}}<M_{\mathrm{D}}$, by e.g., symmetry breaking within a dark sector; $N_{\mathrm{DM}}$ is the DM candidate. In a similar way, a source RHN $N_{\mathrm{S}}$, either $N_{1}$ or $N_{2}$, acquires a mass by coupling to a different scalar field. The important thing is that the dark sector is weakly coupled to the visible sector through a small mixing of the dark RHN $N_{\mathrm{D}}$ with $N_{\mathrm{S}}$ playing the role of a source RHN [2]. This mixing is generated through Higgs portal interactions of $N_{\mathrm{D}}$ with $N_{\mathrm{S}}$ and the SM Higgs $\Phi$, and is described by the operator, $\left(\lambda_{\mathrm{DS}}^{\operatorname{mix}} / \Lambda_{\mathrm{DS}}\right) \Phi^{\dagger} \Phi \overline{N_{\mathrm{D}}^{c}} N_{\mathrm{S}}$ [2-4]. We also introduce a similar operator describing the mixing between the two dark RHNs, $\left(\lambda_{\mathrm{DD}}^{\operatorname{mix}} / \Lambda_{\mathrm{DD}}\right) \Phi^{\dagger} \Phi \overline{N_{\mathrm{D}}^{c}} N_{\mathrm{DM}}$, which is expected to be generated at a different energy scale $\Lambda_{\mathrm{DD}}$ and with a different coupling $\lambda_{\mathrm{DD}}^{\mathrm{mix}}$. For simplicity, we assume that the same new physics generates both operators and that the mixing is the same: $\Lambda_{\mathrm{DS}} / \lambda_{\mathrm{DS}}^{\operatorname{mix}}=\Lambda_{\mathrm{DD}} / \lambda_{\mathrm{DD}}^{\operatorname{mix}}=\tilde{\Lambda}$. The source RHN $N_{\mathrm{S}}$ also has a coupling $\lambda_{\mathrm{S}}$ to the new scalar field $\eta$. Here we do not specify the ultraviolet-complete model that produces such a mixing but the most attractive option is that the same physics is responsible for both $\lambda_{\mathrm{D}(\mathrm{D}, \mathrm{S})}^{\operatorname{mix}}$ and $\lambda_{\mathrm{S}}$. Thus, the SM is extended by

$$
\begin{aligned}
-\mathcal{L}_{\lambda}= & \frac{1}{2} M_{\mathrm{DM}} \overline{N_{\mathrm{DM}}^{c}} N_{\mathrm{DM}}+\frac{1}{2} M_{\mathrm{D}} \overline{N_{\mathrm{D}}^{c}} N_{\mathrm{D}}+\frac{\lambda_{\mathrm{S}}}{2} \eta \overline{N_{\mathrm{S}}^{c}} N_{\mathrm{S}} \\
& +\frac{1}{\tilde{\Lambda}} \Phi^{\dagger} \Phi \overline{N_{\mathrm{D}}^{c}} N_{\mathrm{S}}+\frac{1}{\tilde{\Lambda}} \Phi^{\dagger} \Phi \overline{N_{\mathrm{DM}}^{c}} N_{\mathrm{D}}+\text { H.c.. }
\end{aligned}
$$

The scalar field $\eta$ acquires a vacuum expectation value (VEV) $v_{\eta}$ during a first-order phase transition and, simultaneously, the source RHN acquires a spacetimedependent mass.

Including a fourth RHN, one also recovers the usual type-I seesaw Lagrangian with two RHNs that describes neutrino masses and mixing. At the end of the phase transition the Lagrangian terms extending the SM are (with $I, J=1,2)$

$$
\begin{aligned}
-\mathcal{L}= & \overline{L_{\alpha}} h_{\alpha J} N_{J} \tilde{\Phi}+\frac{1}{2} M_{I} \overline{N_{I}^{c}} \delta_{I J} N_{J} \\
& +\frac{1}{\tilde{\Lambda}} \Phi^{\dagger} \Phi \overline{N_{\mathrm{DM}}^{c}} N_{\mathrm{D}}+\frac{1}{\tilde{\Lambda}} \Phi^{\dagger} \Phi \overline{N_{\mathrm{D}}^{c}} N_{\mathrm{S}} \\
& +\frac{1}{2} M_{\mathrm{DM}} \overline{N_{\mathrm{DM}}^{c}} N_{\mathrm{DM}}+\frac{1}{2} M_{\mathrm{D}} \overline{N_{\mathrm{D}}^{c}} N_{\mathrm{D}}+\text { H.c. },
\end{aligned}
$$

where either $N_{1}$ or $N_{2}$ is identified with the source RHN and the other neutrino is assumed to have negligible or no mixing with the dark neutrinos. Then, the second seesaw RHN plays no role in DM genesis but, in combination with the source RHN, determines neutrino masses and mixing via a two RHN seesaw mechanism which can also potentially generate the matter-antimatter asymmetry via leptogenesis [22].

\section{DARK MATTER GENESIS}

The production of the dark RHN abundance can be calculated by solving the density matrix equation $\left(I, J=N_{D}, N_{S}\right)[5]$

$$
\frac{d N_{I J}}{d t}=-i[\Delta \mathcal{H}, N]_{I J}-\left(\begin{array}{cc}
0 & \Gamma_{\mathrm{dec}} \\
\Gamma_{\mathrm{dec}} & \Gamma_{\mathrm{prod}}
\end{array}\right),
$$

where $N_{I J}$ is the abundance density matrix containing on the diagonal terms the dark and source RHN abundances, normalized in a way that they are simply unity in ultrarelativistic thermal equilibrium. The quantities $\Gamma_{\text {dec }}$ and $\Gamma_{\text {prod }}$ are the decoherence and production rates respectively. The effective Hamiltonian is given by

$$
\Delta \mathcal{H}_{I J} \simeq\left(\begin{array}{cc}
-\frac{\Delta \widetilde{M}^{2}}{4 p} & \Delta H_{\text {mix }} \\
\Delta H_{\text {mix }} & \frac{\Delta \widetilde{M}^{2}}{4 p}
\end{array}\right),
$$

where $\Delta H_{\text {mix }} \equiv T^{2} /(12 \tilde{\Lambda}), \Delta \widetilde{M}^{2} \equiv \widetilde{M}_{\mathrm{S}}^{2}(r, t)-M_{\mathrm{D}}^{2}$, with the $N_{\mathrm{S}}$ effective thermal mass given by the sum of a spacetime-dependent term and thermal terms:

$$
\widetilde{M}_{\mathrm{S}}^{2}(r, t)=M_{\mathrm{S}}^{2}(r, t)+\frac{T^{2}}{4} h_{\mathrm{S}}^{2}+\frac{T^{2}}{8} \lambda_{\mathrm{S}}^{2} N_{N_{\mathrm{S}}} N_{\eta} .
$$

In this expression $h_{\mathrm{S}}^{2} \equiv\left(h^{\dagger} h\right)_{\mathrm{SS}}$ and we introduce the usual effective neutrino mass, $\tilde{m}_{\mathrm{S}}=v^{2} h_{\mathrm{S}}^{2} / M_{\mathrm{S}}$. The seesaw mechanism requires $\tilde{m}_{\mathrm{S}} / m_{\mathrm{sol}} \geq 1$, where $m_{\mathrm{sol}} \simeq 8.6 \mathrm{meV}$ is the solar neutrino mass scale. We take the minimum value $\tilde{m}_{\mathrm{S}} / m_{\text {sol }}=1$ that maximizes the DM lifetime, so that $h_{\mathrm{S}}^{2}=m_{\mathrm{sol}} M_{\mathrm{S}} / v^{2}$.

The spacetime-dependent mass $M_{\mathrm{S}}(r, t)=\lambda_{\mathrm{S}} v_{\eta}(r, t)$ is generated by the VEV of $\eta$ that is described by a wellknown kink solution for the bubble wall profile [23],

$$
v_{\eta}(r, t)=\frac{1}{2} \bar{v}_{\eta}\left[1-\tanh \left(\frac{r-v_{\mathrm{w}}\left(t-t_{\star}\right)}{\Delta_{\mathrm{w}}}\right)\right],
$$

where $v_{\mathrm{w}}$ and $\Delta_{\mathrm{w}}$ are the bubble wall velocity and width. Here, we work in the plasma frame. Note that points in the false vacuum with $v_{\eta}=0$ (outside the bubble), correspond to $r \gg \Delta_{\mathrm{w}}+v_{\mathrm{w}}\left(t-t_{\star}\right)$.

We assume that before the phase transition the $\eta$ abundance $N_{\eta}$ gets thermalized, so that we can set $N_{\eta}=$ $N_{\eta}^{\mathrm{eq}}=N_{\gamma}=4 / 3$ in Eq. (5). On the other hand, the source RHN abundance $N_{N_{\mathrm{S}}}$ is obtained by solving Eq. (3). We adopt a monochromatic approximation with $p \simeq 3 T$. The production rate $\Gamma_{\text {prod }}$ for the $N_{\mathrm{S}}$ abundance is given by the sum of two contributions: $\Gamma_{\mathrm{D}}+\Gamma_{\mathrm{S}}$, from inverse decays 
and scatterings from the Yukawa couplings to the Higgs, and $2 \Gamma_{\eta \rightarrow N_{S} N_{\mathrm{S}}}$, from the decays of $\eta$ into source RHNs. Therefore, explicitly we can write

$$
\frac{d N_{N_{\mathrm{S}}}}{d z} \simeq-(D+S)\left(N_{N_{\mathrm{S}}}-N_{N_{\mathrm{S}}}^{\mathrm{eq}}\right)-D_{\eta} N_{\eta}^{\mathrm{eq}}\left(N_{N_{\mathrm{S}}}^{2}-\left(N_{N_{\mathrm{S}}}^{\mathrm{eq}}\right)^{2}\right),
$$

where we neglected a tiny oscillatory term from the Liouville-von Neumann term from the mixing with $N_{\text {DM }}$. We also introduced the variable $z=T_{\star} / T$, where $T_{\star}$ is a convenient energy scale that we identify with the temperature of the phase transition, and $\left(D, S, D_{\eta}\right) \equiv$ $\left(\Gamma_{\mathrm{D}}, \Gamma_{\mathrm{S}}, 2 \Gamma_{\eta \rightarrow N_{\mathrm{S}} N_{\mathrm{S}}}\right) /(H(z) z)$ with the expansion rate

$$
H(z)=\sqrt{\frac{8 \pi^{3} g_{\star}}{90}} \frac{T_{\star}^{2}}{M_{\mathrm{Pl}}} \frac{1}{z^{2}} \simeq 1.66 \sqrt{g_{\star}} \frac{T_{\star}^{2}}{M_{\mathrm{Pl}}} \frac{1}{z^{2}} .
$$

For $T \gtrsim M_{\mathrm{S}}$ one has $D+S \simeq 2\left(\tilde{m}_{\mathrm{S}} / m_{\mathrm{sol}}\right)\left(M_{\mathrm{S}} / T_{\star}\right)$ $\left(1+\left(8 \pi^{2} / 9\right)\left(M_{\mathrm{S}} / T_{\star}\right) z\right)$. The term from $\eta$ interactions can be written in the form $D_{\eta}=\left(\tilde{m}_{\mathrm{S}}^{\eta} /\left(2 m_{\star}\right)\right)\left(m_{\eta} / T\right)^{2}$, where $m_{\star} \equiv 16 \pi^{5 / 2} \sqrt{g_{\star}} /(3 \sqrt{5})\left(v^{2} / M_{\mathrm{Pl}}\right) \simeq 1.08 \mathrm{meV}$ is the usual equilibrium neutrino mass and, in a similar fashion to Yukawa couplings, we have also introduced the effective neutrino mass $\tilde{m}_{\mathrm{S}}^{\eta} \equiv v^{2} \lambda_{\mathrm{S}}^{2} / T_{\star}$ that parametrizes the coupling of the source RHN to the scalar $\eta$. The scalar $\eta$ has a vanishing or negligible bare mass but it gets a thermal mass $m_{\eta}=g_{\eta} T$, where $g_{\eta}$ is the $\eta$ coupling constant to the thermal bath and typical values are $g_{\eta} \sim 0.1$. For example, for the SM Higgs boson, $m_{H} / T \simeq 0.4$. Henceforth, we set $g_{\eta}=1 / 6$. Since in our case it will turn out that $M_{\mathrm{S}} \simeq T_{\star}$, Yukawa coupling and $\eta$ interactions thermalize the source neutrinos and $N_{N_{\mathrm{S}}} \simeq N_{N_{\mathrm{S}}}^{\mathrm{eq}} \simeq 1$.

We now calculate the dark RHN abundance by solving Eq. (3). This can be done by noticing that before the phase transition, $M_{\mathrm{S}}(r, t)=0$ in Eq. (5). In this way thermal medium effects dominate and suppress the mixing since the off-diagonal term $\Delta H_{\text {mix }}$ is negligible compared to the contribution from $\eta$ interactions in Eq. (5). During the phase transition, the source RHN mass $M_{\mathrm{S}}(r, t)$ increases from zero to its final value $M_{\mathrm{S}}=\lambda_{\mathrm{S}} \bar{v}_{\eta}$. Therefore, during the phase transition, there can be a time when $\Delta \widetilde{M}^{2}\left(r, t_{\text {res }}\right)=0$, or equivalently $\widetilde{M}_{\mathrm{S}}^{2}\left(r, t_{\text {res }}\right)=M_{\mathrm{D}}^{2}$, corresponding to a mixing resonance condition between $N_{\mathrm{S}}$ and $N_{\mathrm{D}}$ :

$$
\frac{M_{\mathrm{D}}^{2}}{M_{\mathrm{S}}^{2}} \simeq\left[\frac{1}{2}-\frac{1}{2} \tanh \left(\frac{r-v_{\mathrm{w}}\left(t_{\mathrm{res}}-t_{\star}\right)}{\Delta_{\mathrm{w}}}\right)\right]^{2}+\frac{T_{\star}^{2}}{6 \bar{v}_{\eta}^{2}},
$$

where we neglected the effective potential generated by the standard Yukawa coupling term since it is much smaller than the term generated by the $\eta$ interactions. Typically, $T_{\star} \sim \bar{v}_{\eta}$ and, for definiteness, we fixed $T_{\star} / \bar{v}_{\eta}=1$. The resonance condition (9) shows that, for given values of $T_{\star}$ and $M_{\mathrm{S}}$,

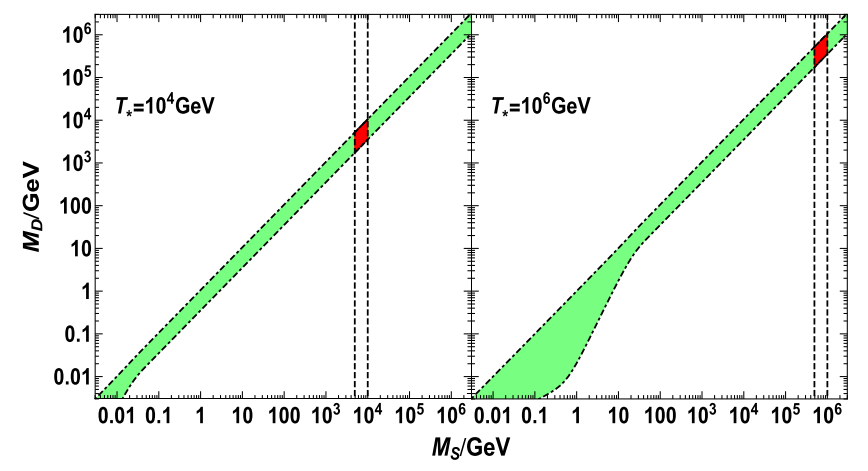

FIG. 1. Dark and source RHN masses that satisfy the resonance condition for two phase transition temperatures. $N_{S}$ are relativistic in the region between the vertical lines.

there is a finite range, $M_{\mathrm{D}}^{\min } \leq M_{\mathrm{D}} \leq M_{\mathrm{D}}^{\max }$, corresponding to a variation of the hyperbolic tangent within $[-1,1]$. For given values of $M_{\mathrm{D}}$ and $M_{\mathrm{S}}$, the resonance can occur either inside or outside the bubble wall, depending on whether $\left|\tanh \left[\left(r-v_{\mathrm{w}}\left(t_{\text {res }}-t_{\star}\right)\right) / \Delta_{\mathrm{w}}\right]\right| \ll 1$ or $\simeq 1$. Therefore, for a fixed value of $T_{\star}$, the resonance condition $9)$ ) identifies an allowed region in the $\left(M_{\mathrm{S}}, M_{\mathrm{D}}\right)$ plane. In Fig. 1 we show such regions for two values of $T_{\star}$. From Eq. (4), if the resonance is crossed quickly enough that the expansion and the variation of the off-diagonal term $\Delta H_{\text {mix }}(t)$ can be neglected during the crossing, and if the decoherence rate $\Gamma_{\mathrm{dec}}=(1 / 2) \Gamma_{\text {prod }}$ is negligible compared to $\left(d \Delta \widetilde{M}^{2}(t) / d t\right)_{\text {res }} /\left(6 T_{\star}\right)$, then the $N_{\mathrm{D}}$ abundance is very well described by the Landau-Zener (LZ) formula $[2,4]$,

$$
\left.N_{N_{\mathrm{D}}}^{\mathrm{res}} \simeq 12 \pi N_{N_{\mathrm{S}}}\left(T_{\star}\right) T_{\star} \frac{\Delta H_{\mathrm{mix}}^{2}}{d \Delta \widetilde{M}^{2} / d t}\right|_{t_{\mathrm{res}}} .
$$

An LZ description was also used in the case of a standard cosmological expansion without a phase transition in Ref. [5]. In that case the bare mass $M_{\mathrm{S}}$ is constant, and for $M_{\mathrm{S}} \gg M_{\mathrm{D}}$, LZ greatly overestimates $N_{\mathrm{D}}$ production because at the resonance, $\Delta M^{2} /(2 E) \ll H$, so that RHN oscillations simply do not have time to develop and LZ breaks down [24]. In our case, $\Delta M^{2} /(2 E) \gg H, v_{\mathrm{w}} / \Delta_{\mathrm{w}}$, must be satisfied to ensure that $N_{S}$ has enough time to oscillate into $N_{D}$. We set $v_{\mathrm{w}} / \Delta_{\mathrm{w}}=T_{\star} / 50$ so that $\mathrm{LZ}$ is valid and the source RHNs are ultrarelativistic at the resonance. This holds for a narrow range, $T_{\star} / 2 \lesssim M_{\mathrm{S}} \lesssim T_{\star}$, between the vertical lines in Fig. 1 for two values of $T_{\star}$. We solved the density matrix equation numerically to verify that $\mathrm{LZ}$ works well within this range, so long as the resonance is also crossed quickly enough. One might consider enhancing DM production by making $\left(d \Delta \widetilde{M}^{2} / d t\right)_{\text {res }}$ smaller, by taking a smaller $v_{\mathrm{w}}$, but too small a value of $v_{\mathrm{w}}$ invalidates LZ which relies on a first-order Taylor expansion about $t_{\text {res }}$. We take $v_{\mathrm{w}}=0.9$ and 0.95 to guarantee the validity of LZ.

We calculate $\left(d \Delta \widetilde{M}^{2} / d t\right)_{\text {res }}$, and arrive at the dark RHN abundance produced at the resonance: 


$$
N_{N_{\mathrm{D}}}^{\mathrm{res}} \simeq \frac{N_{N_{\mathrm{S}}}\left(T_{\star}\right)(\pi / 48)\left(\Delta_{\mathrm{w}} / v_{\mathrm{w}}\right) T_{\star}^{5}}{\tilde{\Lambda}^{2} M_{\mathrm{S}}^{2}\left(r, t_{\mathrm{res}}\right)\left[1-M_{\mathrm{S}}\left(r, t_{\mathrm{res}}\right) / M_{\mathrm{S}}\right]}
$$

However, $N_{\mathrm{D}}$ cannot be DM, since it cannot be long-lived enough to simultaneously satisfy various astrophysics/ cosmology constraints (described below) and reproduce the observed DM abundance, $\Omega_{\mathrm{DM}} h^{2}=0.11933 \pm$ 0.00273 [25] at $3 \sigma$. Indeed, even at zero temperature $N_{D}$ decays because of its mixing with $N_{S}$. We require that $N_{\mathrm{D}}$ dominantly decays to $N_{\mathrm{DM}}$ via $N_{\mathrm{D}} \rightarrow N_{\mathrm{DM}}+2 A$, where $A=W^{ \pm}, Z$, Higgs. We will find its lifetime, $\tau_{\mathrm{D}} \lesssim 10^{-16} \mathrm{~s}$. The $N_{\text {DM }}$ abundance is given by

$$
\Omega_{N_{\mathrm{DM}}} h^{2}=1.0875 \times 10^{6} N_{N_{\mathrm{D}}}^{\mathrm{res}} \frac{M_{\mathrm{DM}}}{\mathrm{GeV}} .
$$

However, $N_{\mathrm{D}}$ can mediate the decay of $N_{\mathrm{DM}}$, thereby placing a lower bound on its lifetime $\tau_{\mathrm{DM}} \sim \tilde{\Lambda}^{2}$, in contrast to the much shorter $N_{\mathrm{D}}$ lifetime which scales as $\tilde{\Lambda}$. For DM masses below $\sim 300 \mathrm{GeV}$, the dominant decay mode is $N_{\mathrm{D}} \rightarrow \nu \ell_{\alpha}^{+} \ell_{\alpha}^{-}(\alpha=e, \mu, \tau)$ with a rate,

$$
\Gamma_{N_{\mathrm{DM}} \rightarrow \nu \ell_{\alpha}^{+} \ell_{\alpha}^{-}}=\frac{\left(\theta_{\Lambda 0}^{\mathrm{D}-\mathrm{S}} \theta_{\Lambda 0}^{\mathrm{DM}-\mathrm{D}}\right)^{2}}{96 \pi^{3}} \frac{\bar{m}_{\alpha}}{M_{\mathrm{S}}} G_{\mathrm{F}}^{2} M_{\mathrm{DM}}^{5},
$$

where $\theta_{\Lambda 0}^{\mathrm{D}-\mathrm{S}(\mathrm{DM}-\mathrm{D})}=2 v^{2} /\left(\tilde{\Lambda}\left(M_{\mathrm{S}(\mathrm{D})}-M_{\mathrm{D}(\mathrm{DM})}\right)\right)$ is the mixing angle between the dark (dark matter) and source (dark) RHNs at zero temperature [4] and $\bar{m}_{\alpha}$ is an effective neutrino mass that depends on $\tilde{m}_{\mathrm{S}}$, an $N_{\mathrm{S}}-\nu_{\alpha}$ Yukawa coupling, and SM gauge couplings. A robust lower bound, $\tau_{\mathrm{DM}} \gtrsim 10^{25} \mathrm{~s}$, is provided by cosmic microwave background (CMB) anisotropy data via changes to the ionization and temperature history [26] (see the Planck labeled black curve in Fig. 2). Data from diffuse $x$-ray and $\gamma$-ray observations also place a lower bound $\tau_{\mathrm{DM}} \gtrsim 10^{25} \mathrm{~s}$ [27] (not shown in Fig. 2) in the range $0.1-10 \mathrm{GeV}$. The points in Fig. 2 correspond to sets of parameter values that reproduce the measured DM abundance and respect the experimental constraints from decays. They are obtained by scanning over $M_{\mathrm{DM}}, M_{\mathrm{S}}, T_{\star}$ (uniform in logarithm) and $\tau_{\mathrm{DM}}$ (instead of $\tilde{\Lambda}$ ). The solutions are divided into five subsets corresponding to the five indicated ranges of $T_{\star}$. There is a tendency for the value of $M_{\mathrm{DM}}$ to increase with $T_{\star}$, which results from the resonance condition (9) that, as we have seen from Fig. 1, imposes a relation between $M_{\mathrm{DM}}, M_{\mathrm{S}}$ and $T_{\star}$.

\section{SPECTRUM OF GRAVITATIONAL WAVES}

We now estimate the potential for current and future $\mathrm{GW}$ interferometers to detect the stochastic GW background produced by the strong first-order cosmological phase transition of $\eta$. We consider the case of nonrunaway bubbles $[28,29]$, in which the scalar field contribution to GWs is ignored, and conservatively assume that only $5 \%$ of the bulk motion of the bubble walls is converted into

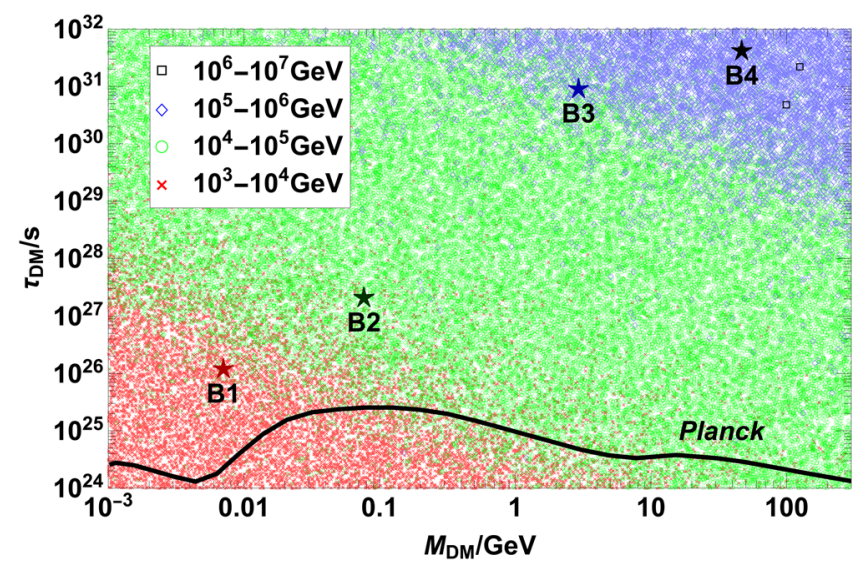

FIG. 2. Points that reproduce the measured DM abundance. All points are generated for $\bar{m}_{\alpha}=0.125 m_{\mathrm{sol}} \simeq 1 \mathrm{meV}, v_{\mathrm{w}} / \Delta_{\mathrm{w}}=$ $T_{\star} / 50$, and for $T_{\star}$ within the five ranges distinguished by the indicated color code. The stars mark four of the five benchmark points in Table I; B5 has a DM lifetime beyond the range of the figure. The black curve indicates the current lower bound on the DM lifetime from Planck data.

vorticity, as supported by numerical results [30]. Then, the contribution from sound waves is the dominant source of the GW background in most of the frequency range. The GW spectrum generated by sound waves during the phase transition is determined by four parameters: the phase transition temperature $T_{\star}$, the bubble wall velocity $v_{\mathrm{w}}$, the ratio of the vacuum energy density released in the transition to the energy density of the radiation bath $\alpha$, and the fraction $\beta / H_{\star}$, where $H_{\star}$ is the expansion rate at $T_{\star}$ and $\beta$ is (approximately) the inverse of the time duration of the phase transition. The last two parameters are irrelevant for DM genesis. We only consider values of $\alpha$ that permit $v_{\mathrm{w}}$ much larger than $c_{s}(1+\sqrt{2 \alpha})$, where $c_{s}=1 / \sqrt{3}$ is the sound speed in the plasma [29]. In this regime the analytical results and fits provided in Ref. [28] can be employed.

In Fig. 3 we display the GW spectrum for our five benchmark points in Table I. Sensitivities of the LIGO O2

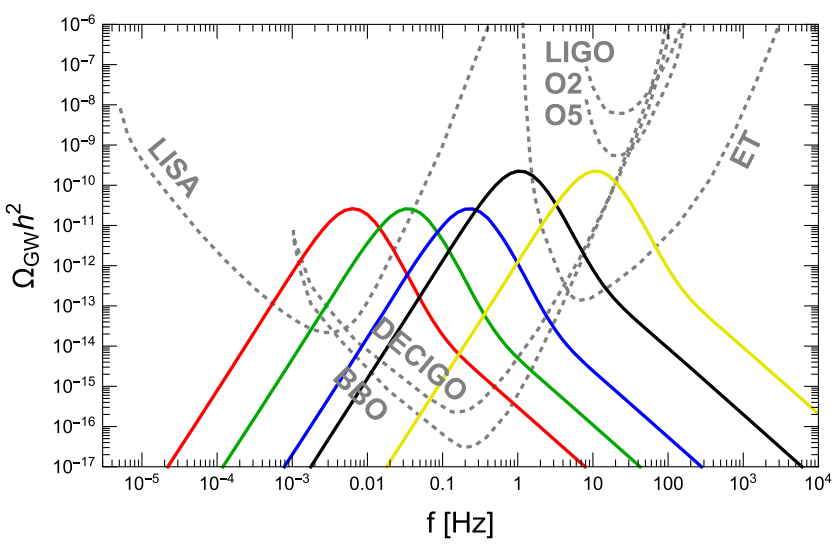

FIG. 3. Predicted spectrum of GWs for the five benchmark points (from left to right) in Table I. 
TABLE I. Benchmark points obtained for $v_{\mathrm{w}} / \Delta_{\mathrm{w}}=T_{\star} / 50$.

\begin{tabular}{lcccccccc}
\hline \hline & $\frac{T_{\star}}{\mathrm{PeV}}$ & $\frac{\tau_{\mathrm{DM}}}{10^{26} \mathrm{~s}}$ & $\frac{M_{\mathrm{S}}}{\mathrm{TeV}}$ & $\frac{M_{\mathrm{D}}}{\mathrm{TeV}}$ & $\frac{M_{\mathrm{DM}}}{\mathrm{GeV}}$ & $v_{\mathrm{W}}$ & $\alpha$ & $\frac{\beta}{H_{\star}}$ \\
\hline B1 & $3 \times 10^{-3}$ & 1.219 & 1.57 & 0.567 & $7 \times 10^{-3}$ & 0.90 & 0.10 & 10 \\
B2 & 0.016 & 21.26 & 12.9 & 7.72 & 0.077 & 0.90 & 0.10 & 10 \\
B3 & 0.106 & $9.25 \times 10^{4}$ & 93.3 & 72.6 & 2.92 & 0.90 & 0.10 & 10 \\
B4 & 1.052 & $4.24 \times 10^{5}$ & 666 & 666 & 46.69 & 0.95 & 0.15 & 5 \\
B5 & 10.75 & $4.69 \times 10^{17}$ & $8.7 \times 10^{3}$ & $5.3 \times 10^{3}$ & 175.8 & 0.95 & 0.15 & 5 \\
\hline \hline
\end{tabular}

and O5 observing runs [31], LISA [28,32], ET [33], BBO [34] and DECIGO [35] are shown for comparison. TianQin [36] and Taiji [37] have sensitivities similar to LISA. As shown in Fig. 2, successful DM genesis requires $T_{\star} \gtrsim$ $10^{4} \mathrm{GeV}$ for the bulk of the points. LISA can test this regime. LIGO, ET, BBO and DECIGO might be able to test a high-temperature phase transition, $T_{\star} \sim 10^{7}-10^{8} \mathrm{GeV}$, if $\alpha$ takes a relatively large value. For a five-year LISA mission with a $75 \%$ duty cycle, we find the signal-to-noise ratio for $\mathrm{B} 1$-B5 to be $\mathcal{O}\left(10^{5}, 10^{4}, 10^{2}, 10,10^{-2}\right)$, respectively. Since in all cases $M_{\mathrm{S}}$ is larger than $300 \mathrm{GeV}$, leptogenesis is viable.

\section{FINAL REMARKS}

We introduced a novel model in which DM and neutrino masses are generated during a phase transition of a scalar field. The phase transition needs to be strongly first order for efficient DM genesis, which has the welcome feature of the associated production of GWs with an intensity measurable at the planned GW interferometers, LISA, BBO, DECIGO, ET, TianQin and Taiji. We find solutions for DM masses within a range $1 \mathrm{MeV}-300 \mathrm{GeV}$ with a clear tendency of the temperature of the phase transition to increase with the dark matter mass, though the dependence also involves other parameters such as the mass of the source RHN. Interestingly, our solutions have source RHNs heavier than $300 \mathrm{GeV}$ which allows successful resonant leptogenesis [38] with two RHNs [5]. We have also seen that the decays of the dark RHNs offer an additional way to test the scenario through observations of CMB anisotropies and/or the diffuse x-ray and $\gamma$-ray backgrounds. We have not thoroughly studied the dependence of our results on all parameters and have fixed some of them to reasonable values, e.g., $g_{\eta}=1 / 6, \quad \bar{v}_{\eta}=T_{\star}$ and $\quad \tilde{m}_{\mathrm{S}} / m_{\mathrm{sol}}=1$.
However, we verified that our results are insensitive to small variations of the first two parameters. We restricted ourselves to values of $M_{\mathrm{S}} \simeq T_{\star}$ so as to use the $\mathrm{LZ}$ approximation. Numerical density matrix calculations should determine the full range of allowed values for $M_{\mathrm{S}} / T_{\star}$. We also assumed a specific bubble wall profile with a fixed value of the bubble width and velocity. A thorough exploration of the allowed values of all parameters, including the possibility of DM masses above $300 \mathrm{GeV}$, will be presented in a forthcoming paper together with some variants of the model [39]. However, our results clearly prove the viability of the proposed mechanism and how DM genesis, although escaping traditional direct and collider searches, will be tested in the future with cosmology, cosmic rays and GWs.

\section{ACKNOWLEDGMENTS}

We thank L. Bian, Z. Liu, K. Farrag and R. Samanta, for useful discussions. D. M. thanks the University of Southampton and the Aspen Center for Physics (which is supported by U.S. National Science Foundation (NSF) Grant No. PHY-1607611) for their hospitality while this work was in progress. P. D. B. thanks Institute for Nuclear Theory (INT) at the University of Washington for its hospitality and the U.S. Department of Energy (DOE) for partial support during the completion of this work. P. D. B. and Y.L.Z. acknowledge financial support from the Science and Technology Facilities Council (STFC) Consolidated Grant No. L000296/1. This project has received funding/support from the European Union Horizon 2020 research and innovation programme under the Marie Skłodowska-Curie Grant agreements No. 690575 and 674896. D. M. is supported in part by U.S. DOE Grant No. de-sc0010504.
[1] G. Bertone and D. Hooper, Rev. Mod. Phys. 90, 045002 (2018); P. Di Bari, Cosmology and the Early Universe (CRC Press, Boca Raton, FL, 2018).

[2] A. Anisimov and P. Di Bari, Phys. Rev. D 80, 073017 (2009).
[3] A. Anisimov, Majorana dark matter, The Identification of Dark Matter (World Scientific, Singapore, 2007), pp. 439449.

[4] P. Di Bari, P. O. Ludl, and S. Palomares-Ruiz, J. Cosmol. Astropart. Phys. 11 (2016) 044. 
[5] P. Di Bari, K. Farrag, R. Samanta, and Y. L. Zhou, J. Cosmol. Astropart. Phys. 10 (2020) 029.

[6] S. Pascoli, J. Turner, and Y. L. Zhou, Phys. Lett. B 780, 313 (2018).

[7] E. Witten, Phys. Rev. D 30, 272 (1984).

[8] C. Hogan, Mon. Not. R. Astron. Soc. 218, 629 (1986).

[9] M. Turner and F. Wilczek, Phys. Rev. Lett. 66, 5 (1991).

[10] M. Kamionkowski, A. Kosowsky, and M. S. Turner, Phys. Rev. D 49, 2837 (1994).

[11] T. Cohen, D. E. Morrissey, and A. Pierce, Phys. Rev. D 78, 111701 (2008).

[12] A. Falkowski and J. M. No, J. High Energy Phys. 02 (2013) 034.

[13] K. Tsumura, M. Yamada, and Y. Yamaguchi, J. Cosmol. Astropart. Phys. 07 (2017) 044.

[14] F. P. Huang and C. S. Li, Phys. Rev. D 96, 095028 (2017).

[15] L. Bian and Y. L. Tang, J. High Energy Phys. 12 (2018) 006.

[16] Y. Bai, A. J. Long, and S. Lu, Phys. Rev. D 99, 055047 (2019).

[17] M. Breitbach, J. Kopp, E. Madge, T. Opferkuch, and P. Schwaller, J. Cosmol. Astropart. Phys. 07 (2019) 007.

[18] E. Hall, T. Konstandin, R. McGehee, and H. Murayama, arXiv:1911.12342.

[19] M. Baker, J. Kopp, and A. Long, Phys. Rev. Lett. 125, 151102 (2020).

[20] D. Chway, T. H. Jung, and C. S. Shin, Phys. Rev. D 101, 095019 (2020).

[21] D. Marfatia and P. Y. Tseng, arXiv:2006.07313.
[22] M. Fukugita and T. Yanagida, Phys. Lett. B 174, 45 (1986).

[23] P. John, Phys. Lett. B 452, 221 (1999).

[24] P. Di Bari, R. Samanta, and Y. L. Zhou (to be published).

[25] Y. Akrami et al. (Planck Collaboration), Astron. Astrophys. 641, A1 (2020).

[26] T. R. Slatyer and C. L. Wu, Phys. Rev. D 95, 023010 (2017).

[27] R. Essig, E. Kuflik, S. McDermott, T. Volansky, and K. Zurek, J. High Energy Phys. 11 (2013) 193.

[28] C. Caprini et al., J. Cosmol. Astropart. Phys. 04 (2016) 001.

[29] C. Caprini et al., J. Cosmol. Astropart. Phys. 03 (2020) 024.

[30] M. Hindmarsh, S. J. Huber, K. Rummukainen, and D. J. Weir, Phys. Rev. D 92, 123009 (2015).

[31] B. P. Abbott et al. (KAGRA, LIGO Scientifi,c and Virgo Collaborations), Living Rev. Relativity 21, 3 (2018).

[32] P. Auclair et al., J. Cosmol. Astropart. Phys. 04 (2020) 034.

[33] S. Hild et al., Classical Quantum Gravity 28, 094013 (2011).

[34] K. Yagi and N. Seto, Phys. Rev. D 83, 044011 (2011); 95, 109901(E) (2017).

[35] S. Kawamura et al. (DECIGO Collaboration), Proc. Sci., KMI2019 (2019) 019.

[36] J. Luo et al. (TianQin Collaboration), Classical Quantum Gravity 33, 035010 (2016).

[37] W. H. Ruan, Z. K. Guo, R. G. Cai, and Y. Z. Zhang, Int. J. Mod. Phys. A 35, 2050075 (2020).

[38] A. Pilaftsis and T. E. J. Underwood, Nucl. Phys. B692, 303 (2004).

[39] P. Di Bari, D. Marfatia, and Y. L. Zhou (to be published). 